УДК 621.396 .2

P.І. Чорнобай, Д.А. Маковєєнко

ДП Український НДІ Радіо і телебачення, вул. Буніна, 31, Одеса, 65025

\title{
АНАЛІЗ ПІДВИЩЕННЯ ПРОПУСКНОЇ ЗДАТНОСТІ ПРИ ВИКОРИСТАННІ SFR В МЕРЕЖІ Е- UTRA
}

У статті проведено аналіз підвищення пропускної здатності мережі E-UTRA при використанні м'якого частотного перевикористання. Представлена методика розрахунку пропускної здатності мережі мобільного широкосмугового радіодоступу LTE при використанні SFR і режиму адаптації параметрів MCS до середовища розповсюдження.

Ключові слова: LTE - MCS - SFR - пропускна здатність.

Р.И. Чернобай, Д.А. Маковеенко

ГП Украинский НИИ Радио и телевидения, ул. Бунина, 31, Одесса, 65025

\section{АНАЛИЗ ПОВЫШЕНИЯ ПРОПУСКНОЙ СПОСОБНОСТИ ПРИ ИСПОЛЬЗОВАНИИ SFR В СЕТИ E-UTRA}

В статье проведен анализ повышения пропускной способности сети E-UTRA при использовании мягкого частотного переиспользования (SFR). Представлена методика расчета пропускной способности сети мобильного широкополосного радиодоступа LTE при использовании SFR и режима адаптации параметров модуляционно-кодовых схем (MCS) к среде распространения.

Ключевые слова: LTE - MCS - SFR - пропускная способность.

\section{P.I. Chernobay, D.A. Makoveyenko}

Ukrainian Scientific Research Institute of Radio and TV, 31 Bunina str., Odessa, 65025

\section{ANALYSIS OF INCREASE CAPACITY E-UTRA NETWORK USING SFR}

The article analyzes the increase capacity E-UTRA network using soft frequency reuse. The technique of network bandwidth mobile broadband wireless LTE using SFR and adaptation mode parameters modulation-coding schemes to the radio wave propagation.

Keywords: LTE - MCS -SFR - capacity.

\section{I. ВВЕДЕНИЕ}

В сети мобильного широкополосного беспроводного доступа (ШБД) E-UTRA (3GPP LTE) используется мультиплексирование с ортогональным частотным разделением каналов (OFDM) [1] Метод доступа с ортогональными поднесущими OFDMA является расширением OFDM для обеспечения множественного доступа к сети. OFDMA распределяет поднесущие между пользователями и, таким образом, позволяет одновременное использование радиочастотного ресурса несколькими абонентами на приемной стороне [2].

Одной из важнейших проблем развертывания сетей мобильной связи является минимизация используемого частотного ресурса при обеспечении внутрисистемной электромагнитной совместимости (ЭМС). Традиционное [3] ЧТП планирование заключается в выборе коэффициента повторного использования частот (размерности кластера), обеспечивающему отсутствие помех на краю соты, в совмещенном частотном радиоканале.

Метод доступа OFDM открывает новые возможности при частотно-территориальном планирования (ЧТП) сетей мобильной связи. При планировании сети, использующей метод OFDM, по- лоса частот разделяется на группы поднесущих с различной мощностью. Одним из вариантов такого разделения на группы поднесущих является мягкое частотное переиспользование (SFR), которое должно существенно повысить пропускную способность сети, при работе совместно с режимом адаптации параметров модуляционно-кодовых схем.

Целью данной статьи является анализ повышения пропускной способности сети E-UTRA при использовании мягкого частотного переиспользования (ЧП).

\section{II. ЧТП ПРИ МЯГКОМ ЧАСТОТНОМ ПЕ- РЕИСПОЛЬЗОВАНИИ}

Коэффициент переиспользования частот количество ячеек, которые могут использовать одинаковый частотный ресурс $\left(f_{r}\right)$. Будем различать три основных схемы частотного переиспользования: полное, статическое и мягкое.

При полном частотном переиспользовании распределение частот между соседними базовыми станциями (БС) отсутствует $\left(f_{r}=1\right)$. 
Главным недостатком данной схемы является то, что абонентские устройства которые находятся на границе ячейки попадают под влияние помех, создаваемых каналами соседних ячеек, что значительно влияет на общую производительность системы и снижает скорость передачи данных. Обычно такая схема не используется.

Схема статичного ЧП в основном используется в многоканальных системах связи, использующих частотное и/или временное разделение каналов. Основной идеей данной схемы является то, что все частотные каналы разделяются на $f_{r}=3,4,7$ или 9 (в зависимости от критериев обеспечения внутрисистемной ЭМС). Эти наборы каналов присваиваются отдельной БС, таким образом что соседние ячейки не используют одинаковые частоты. Пример схемы со статическим частотным переиспользования при $f_{r}=3$ приведен на рисунке 1 .

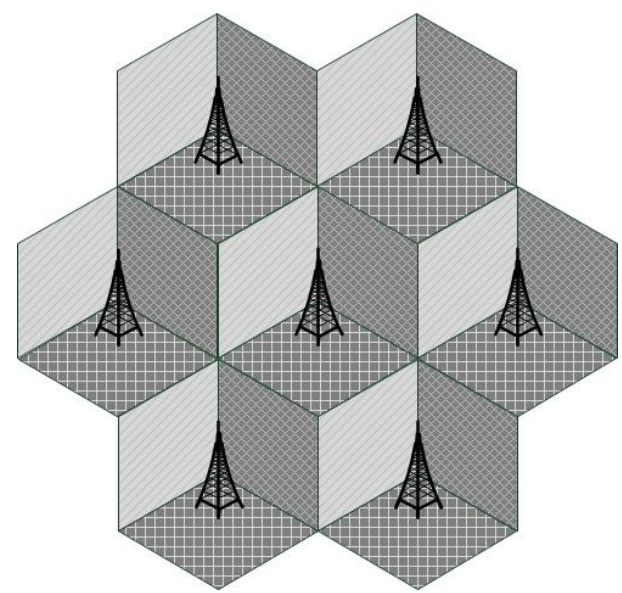

Рисунок 1 - Схема со статичным ЧП и коэффициентом переиспользования три

Использование данной схемы значительно снижает внутрисетевого помеху для любой пары ячеек, в отличие от схемы с общим частотным переиспользованием.

Однако при таком частотном распределения общая пропускная способность системы уменьшается в $f_{r}$ раз:

$$
C=\frac{W}{f_{r}} \log _{2}(1+\operatorname{SINR}),
$$

где $C$ - пропускная способность в радиоканале, Мбит/c; $W$ - ширина канала, выделенного для развертывания сети, МГц; SINR - отношение сигнал/(помеха+шум), дБ.

При планировании сети с помощью данных схемы мягкого частотного переиспользования общая полоса частот разделяется на группы поднесущих с различной мощностью (рис. 2).

Группа поднесущих с максимальной мощностью называется основной группой, а поднесущие c меньшей мощностью называются вторичной группой. Для вторичной группы водится ограни- чение мощности, определяемое коэффициентом соотношения мощности, меняющимся от 0 до 1.

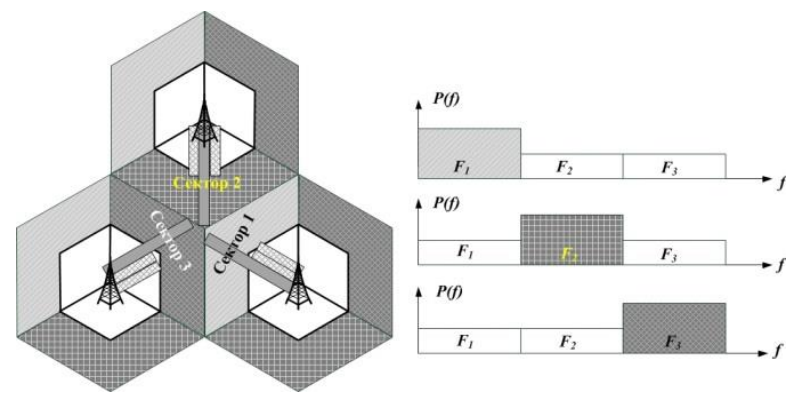

\section{Рисунок 2 - Схема мягкого частотного переиспользования}

Особенностью такой схемы является то, что пользователям на границе ячейки назначается коэффициент переиспользования, равный трем, а пользователям в центре ячейки назначается коэффициент переиспользования, равный одному.

\section{III. МОДЕЛЬ РАСЧЕТА ПРОПУСКНОЙ СПОСОБНОСТИ СЕТИ Е-UTRA ПРИ ИС- ПОЛЬЗОВАНИИ SFR.}

Для анализа пропускной способности сети ЕUTRA рассмотрим гексагональную систему координат, в узлах которой расположены базовые станции.

Отношение сигнал/(помеха+шум) для схемы со статичным ЧП определяется как:

$$
\operatorname{SINR}=\frac{P_{r x}}{P_{n}+P_{\text {out }}},
$$

где $P_{r x}$ - уровень сигнала на входе приемника мобильной станции; $P_{n}-$ уровень собственных шумов; $P_{\text {out }}-$ уровень внутрисистемной помехи на входе мобильной станции от базовых станций в совмещенном канале приема.

При отдалении мобильной станции от базовой значение при помощи обратной связи контролируется значение SINR и через индикаторы качества канала изменяются параметры передачи и модуляционно-кодовая схема. Таким образом, определив значение сигнал/(помеха+шум) можно найти пропускную способность радиоканала. Если абоненты размещены равномерно в зоне обслуживания базовой станции, средняя спектральная эффективность ячейки будет определяться усреднением площади, в которой работает данная MCS на её спектральную эффективность:

$$
\bar{C}=\frac{\sum_{i}^{N} S_{i} \cdot C_{i}}{S_{N}},
$$

где $\bar{C}$ - среднее значение спектральной эффективности, бит/с/Гц; $S_{i}-$ площадь зоны покрытия, 
соответствующая $i$-ой $\operatorname{SINR} ; S_{N}$ - площадь зоны покрытия соты.

Площадь сегментов с $i$-ым значением SINR можно рассчитать из формулы:

$$
S_{i}=\pi r_{i}^{2}-\pi\left(r_{i-1}\right)^{2},
$$

где $r_{i}$ - радиус зоны покрытия, соответствующий $i$-ой SINR .

Для схемы мягкого ЧП необходимо рассматривать отношение SINR отдельно для основной и вторичной групп несущих (рис. 3).

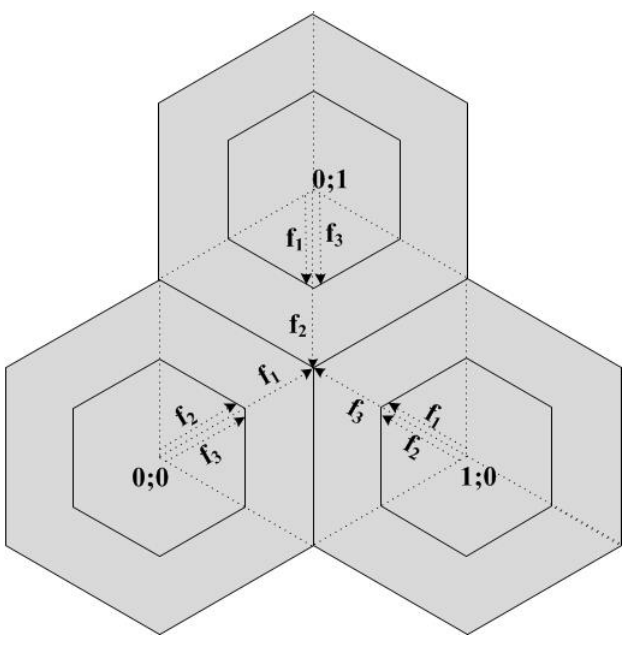

Рисунок 3 - Расчет помехи для схемы SFR

Так для основной группы поднесущих:

$$
\operatorname{SINR}_{m}=\frac{P_{r x}}{P_{n}+P_{\text {out }}}=\frac{P_{r x}}{P_{n} \cdot k+P_{\text {out }}+P_{\text {in }} \cdot m},
$$

где $P_{i n}-$ уровень внутрисистемной помехи на входе мобильной станции на частотах вторичной группы; $P_{\text {out }}$ - уровень внутрисистемной помехи на входе мобильной станции на частотах основной группы; $k$ - соотношение несущих основной группы ко всем несущим; $m$ - коэффициент соотношения мощности несущих вторичной группы к мощности несущих основной группы. Пропускная способность для основной группы поднесущих снижается в $k$ раз:

$$
C_{m}=\frac{W}{k} \log _{2}\left(1+\operatorname{SINR}_{m}\right),
$$

Так вторичной группы поднесущих:

$$
\operatorname{SINR}_{v}=\frac{P_{r x}}{P_{n}+P_{\text {out }}}=\frac{P_{r x} \cdot m}{P_{n} \cdot(1-k)+P_{\text {out }} \cdot(m+1)+P_{\text {in }} \cdot m}
$$

Пропускная способность для вторичной группы поднесущих снижается в $(1-k)$ раз:

$$
C_{v}=\frac{W}{1-k} \log _{2}\left(1+\operatorname{SINR}_{v}\right),
$$

Общая пропускная способность в схеме SFR определяется суммой пропускной способности первичной и вторичной групп поднесущих.

Для сравнения пропускной способности схемы статичного ЧП при $f_{r}=3$ и схемы SFR проведем моделирование помех для сети из семи базовых станций. В такой сети учитываются помехи от первого круга, которые составляют до $90 \%$ от общего уровня помехи. Параметры и требования к канальному и системному уровням сети E-UTRA приведены в Таблице 1.

Таблица 1 - Исходные параметры и требования

\begin{tabular}{|l|c|}
\hline \multicolumn{1}{|c|}{ Параметр } & Значение \\
\hline \multicolumn{2}{|c|}{ Кехьй уровень } \\
\hline $\begin{array}{l}\text { Технология множественных ан- } \\
\text { тенн }\end{array}$ & $\begin{array}{c}\text { SISO (один } \\
\text { вход - один } \\
\text { выход) }\end{array}$ \\
\hline Модель радиоканала канала & Cost 231 \\
\hline Высота БС & 40 м \\
\hline Высота АС & 1,5 м \\
\hline \multicolumn{2}{|c|}{ Састй уровень } \\
\hline Частота несущей БС & 1850 МГц \\
\hline Ширина полосы канала & 15 МГц \\
\hline Мощность передатчика БС & 20 ВТ \\
\hline Усиление антенны & 15 дБи \\
\hline Потери в фидерном тракте & 2 дБ \\
\hline Потери проникновения в здание & 10 дБ \\
\hline Запас на медленные замирания & 5 дБ \\
\hline Удельная мощность шума & -174 дБм/Гц \\
\hline Собственный шум приемника & 6 дБ \\
\hline $\begin{array}{l}\text { Отношение мощности вторичной } \\
\text { группы к мощности основной } \\
\text { группы }\end{array}$ & $1 / 2$ \\
\hline $\begin{array}{l}\text { Отношение количества несущих } \\
\text { основной группы ко всем несущим }\end{array}$ & $1 / 3$ \\
\hline
\end{tabular}

\section{IV. КОЛИЧЕСТВЕННЫЕ ОЦЕНКИ ПРО- ПУСКНОЙ СПОСОБНОСТИ СЕТИ Е-UTRA ПРИ ИСПОЛЬЗОВАНИИ SFR.}

Рассмотрим ячейку, радиусом 1 км, разделенную равномерно на 10 подзон. В каждой из подзон рассчитывается значение SINR, используя расширенную модель Хата [4-5] для исходных данных, представленных в таблице 1.

Исходя из (1) можно найти пропускную способность для схемы со статичным ЧП и коэффициентом переиспользования три.

Для схемы мягкого частотного переиспользования отдельно определяется SINR для первичной и вторичной групп поднесущих. Далее в соответствии с (6-7) можно найти пропускную способность для основной и вторичной групп поднесущих. схемы со статичным ЧП и коэффициентом переиспользования три. При моделировании пропускной способности SFR принято, что отношение мощности поднесущих равно половине к мощности основной группы, а количество поднесущих 
основной группы в три раза меньше чем количество всех несущих.

Количественные оценки моделирования пропускной способности в секторе сети E-UTRA при использовании схемы со статичным ЧП $\left(f_{r}=3\right)$ и SFR приведены на рисунке 4.

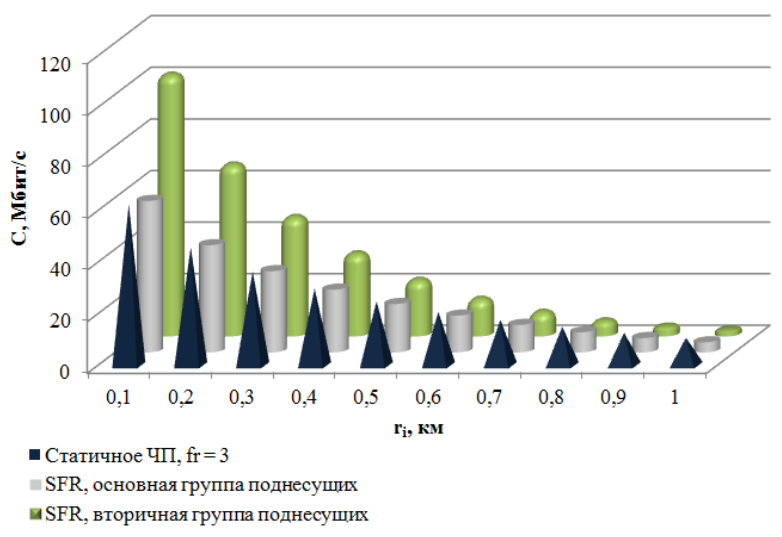

Рисунок 4 - Схема мягкого частотного
переиспользования

Как видно из рисунка 4 на небольших расстояний от базовой станции (до 400 м) наибольшая пропускная способность достигается для вторичной группы поднесущих в режиме SFR. Это объясняется тем, что отношение количества несущих вторичной группы ко всем несущим составляет $2 / 3$ а на небольших расстояниях от базовой станции отношение SINR составляет до 35 дБ. При увеличении расстояния между мобильной и базовой станцией пропускная способность сети уменьшается для расстояний более 600 м больший вклад в пропускную способность схемы SFR вносит основная группа поднесущих. Наибольший интерес представляет усредненная пропускная способность соты, полученная используя выражение (3) для равномерного расположения абонентов внутри соты. Полученная в результате моделирования, усредненная пропускная способность в одном секторе составляет:

- для схемы со статичным ЧП и коэффициентом переиспользования 19,18 Мбит/с;

- для основной группы поднесущих в схеме SFR 12,61 Мбит/с;

- для основной группы поднесущих в схеме SFR 14,37 Мбит/с.

Таким образом, общая пропускная способность режима SFR составляет 26,98 Мбит/с, что на 40,7\% больше, чем пропускная способность сектора, использующего схему статичного частотного переиспользования.

\section{V. ЗАКЛЮЧЕНИЕ}

В статье представлена модель расчета пропускной способности сети E-UTRA при использовании SFR. Отличием схемы мягкого частотного переиспользования является то, что пользователям на границе ячейки назначается коэффициент пере- использования, равный трем, а пользователям в центре ячейки назначается коэффициент переиспользования, равный одному. Это возможно благодаря использованию метода OFDM и разделению полоса частот на группы поднесущих с различным уровнем мощности. Количественные оценки сравнения пропускной способности сети с мягким и статичным ЧП показывают, что общая пропускная способность режима SFR составляет 26,98 Мбит/с, что на 40,7\% больше, чем пропускная способность сектора, использующего схему статичного частотного переиспользования.

Дальнейшее совершенствование метода SFR связано с выбором оптимальных соотношений мощности вторичной группы к мощности основной группы, а также количества несущих основной группы ко всем несущим.

\section{ЛИТЕРАТУРА}

1. Маковеенко Д.А. Исследование особенностей физического уровня в стандарте LTE / Д.А. Маковеенко, А.С. Степанцов - Холодильна техніка і технологія. - 2011. - № 5. - С.86 - 90.

2. Тихвинский В.О. Сети мобильной связи LTE. Технологии и архитектура / В.О. Тихвинский, С. В. Терентьев, А. Б. Юрчук - М.: Эко-Трендз, 2010. $-284 \mathrm{c}$

3. Бабков В.Ю. Сети мобильной связи. Частотнотерриториальное планирование / В.Ю. Бабков, Вознюк М.А., Михайлов П.А. - М.: Горячая линия-Телеком, 2007. - 224 с.

4. Стапанцов А.С. Методы повышение спектральной эффективности в стандарте LTE / A.C. Степанцов, Д.А. Маковеенко - Холодильна техніка і технологія. - 2011. - № 6. - С.92 - 96

5. Report ITU-R SM.2028-1 Monte Carlo simulation methodology for the use in sharing and compatibility studies between different radio services or systems 2001, 2002.

\section{REFERENCES}

1. Makoveenko D.A. Issledovanie osobennostej fizicheskogo urovnja v standarte LTE / D.A. Makoveenko, A.S. Stepancov - Holodilna tehnika i tehnologija. - 2011. - № 5. - S. 86 - 90 .

2. Tihvinskij V.O. Seti mobilnoj svjazi LTE. Tehnologii i arhitektura / V.O. Tihvinskij, S. V. Terent'ev, A. B. JUrchuk - M.: JEko-Trendz, 2010. $284 \mathrm{~s}$.

3. Babkov V.U. Seti mobilnoj svjazi. CHa-stotnoterritorialnoe planirovanie / V.U. Babkov, Voznjuk M.A., Mihajlov P.A. - M.: Gorjachaja linija-Telekom, 2007. - $224 \mathrm{~s}$.

4. Stepancov A.S. Metody povyshenie spektralnoj jeffektivnosti v standarte LTE / A.S. Stepancov, D.A. Makoveenko - Holodilna tehnika i tehnologija. 2011. - № 6. - S. $92-96$

5. Report ITU-R SM.2028-1 Monte Carlo simulation methodology for the use in sharing and compatibility studies between different radio services or systems 2001, 2002. 


\section{ANALYSIS OF THROUGHPUT INCREASE WHEN USING SFR IN THE E-UTRA NETWORK}

This article focuses on the analysis of E-UTRA network throughput possible increase using soft frequency reuse (SFR) for orthogonal frequency division multiplexing (OFDM). The first part describes the circuit of soft frequency reuse, a peculiarity of which is that on the border of the cell reusability factor is three and one in the center of the cell. In the second part for the for the analysis of network bandwidth E-UTRA the analytical model of calculation is given, based on the determination of the signal/(interference+noise) ratio when removing from the base station, taking into consideration the channel quality indicators and changes in modulation-coding schemes parameters. The third part presents quantitative estimation of the network E-UTRA throughput ability at the use SFR scheme. For comparison of the throughput ability static frequency reusability and SFR schemes the simulation of the interferences for network from seven base stations are given. Parameters and requirements for channel and system levels correspond to the 3GPP requirements to network E-UTRA. The simulation found that the network throughput with a soft SFR is on 40.7\% higher than with static and is $26.98 \mathrm{Mbit} / \mathrm{s}$ in one operation sector of a base station for a channel bandwidth of $15 \mathrm{MHz}$.

Keywords: LTE - MCS-SFR - capacity.

Отримана в редакції 09.01.2014, прийнята до друку 04.03.2014 